\title{
Improving detection of alcohol misuse in patients presenting to an accident and emergency department
}

\author{
J S Huntley, C Blain, S Hood, R Touquet
}

\begin{abstract}
Objectives-To assess and improve deployment of a brief test for alcohol misuse: the Paddington Alcohol Test (PAT).

Design-Prospective study of the effects of audit feedback.

Setting-An urban accident and emergency department.

Subjects-Senior house officers (SHO) (n = 13).

Outcome measurements-PAT use and categorisation of patients for each SHO; observational analysis of presenting complaints according to PAT.

Results-1062 of 1737 patients $(61.1 \%)$ were defined as PAT possible-that is, presented with $\geqslant$ one complaint listed on the PAT test proforma. In month 1 , PAT uptake was poor. PAT use improved significantly when feedback was instituted $(p<0.0001)$. The response to audit and feedback showed marked inter-SHO variation. When feedback was withdrawn (month 4 ), there was a significant reduction in PAT use $(p=0.003)$. Three other indices of detection followed this trend: (a) number of PAT positive patients identified, (b) proportion of PAT possible patients identified as PAT positive, and (c) number of PAT positive patients referred to the alcoholic health worker. The 10 most common PAT positive categories, accounting for $77 \%$ of all PAT positive complaints were: fall > collapse (including "fit", "blackout") > head injury (including "facial injury") > assault (including "domestic violence" and 'other') > nonspecific gastrointestinal problem > "unwell" > psychiatric (including "depression", "overdose", "confusion") > cardiac (including "chest pain" and "palpitations") > self neglect > repeat attendance. Conclusions-Ongoing audit with feedback improves both PAT use and detection of alcohol misuse. The PAT is now simpler including only 10 conditions, which should further aid its use.

(Emerg Med F 2001;18:99-104)
\end{abstract}

Keywords: alcohol misuse; alcohol health worker; Paddington Alcohol Test

Alcohol misuse is a major cause of mortality and morbidity throughout the UK, ${ }^{12}$ whether this be directly (pathophysiological, for example: intoxication, hypertension, cirrhosis, cancers) or indirectly (via social harm, for example: road accidents, drownings, child abuse, murders, other assaults, deaths by fire). ${ }^{3-11}$ The problem is exacerbated by the alcohol accepting culture of the Western world, stigmatisation of the alcoholic and apathy or lack of skills on the part of the doctor, ${ }^{12-14} \mathrm{com}-$ pounded by denial on the part of the patient.

There are few opportunities for alcohol detection, ${ }^{15}$ but the accident and emergency department $(\mathrm{A} \& \mathrm{E})$ is in a potentially pivotal position to detect and refer patients who misuse alcohol. ${ }^{11}{ }^{16-18}$ More patients attend A\&E than outpatient departments. ${ }^{19}$ Attendance at $A \& E$ is often for a crisis when patients are more willing to accept help. ${ }^{20}$ Many of these patients also suffer from the effects of acute or chronic alcohol misuse, or both. ${ }^{21-23}$ In our area of London (Kensington, Chelsea and Westminster) it has been estimated that $22.2 \%$ of people drink in excess of recommended values, compared with $10 \%$ nationally. ${ }^{24}$

In the USA the subject of patients with alcohol problems in A\&E has been extensively reviewed, ${ }^{25}{ }^{26}$ the conclusions being very similar to those in the UK: "the ED is an excellent site for brief intervention with problem drinkers; patients are often more receptive to education in the moment of crisis". However, compared with the Paddington Alcohol Test (PAT) (three questions), the questionnaires used in the USA are either more comprehensive-thus taking longer to complete-for example, TWEAK, AUDIT, Brief MAST, or if as short, such as the four question CAGE, detect only those who are, or who are becoming, dependent on alcohol and not those who are intermittent binge drinkers - that is, hazardous drinkers.

The effectiveness of brief motivational interventions in the treatment of alcohol misuse in patients admitted with an injury was confirmed in a prospective randomised study carried out in a US trauma centre. ${ }^{27} \mathrm{~A}$ total of 2524 patients were screened of whom 1153 (46\%) met the criteria for alcohol misuse - a figure close to that found in UK studies. ${ }^{28}{ }^{29}$ Of these, 396 were allocated to the control group, and 366 to the intervention group. Of the 391 not entered in the trial, the refusal rate was only $18.6 \%$ (215 patients); the other 176 screened positive but were discharged over the weekend (study staff were not available to enter patients into the programme). In the intervention group, alcohol consumption and trauma recurrence were both significantly reduced. As alcohol misuse is the leading risk factor for injury, this study provides further evidence for the efficacy of an A\&E based alcohol screening and referral system such as that based on the PAT.

Cherpitel (1993) reviewed international emergency room studies concerning the 
relationship between alcohol and injuries, and concluded that further research was needed about patients reattending with alcohol related accidents. ${ }^{30}$ A recent UK survey of $A \& E$ departments ${ }^{31}$ showed that the general attitude of doctors and nurses towards improving the response to alcohol related attendances was positive. The association with alcohol misuse is well documented for some frequent A\&E presentations, for example, assault and head injury-especially facial injury. ${ }^{62}$ Despite this, few $A \& E$ doctors routinely inquire about or record alcohol consumption. ${ }^{31}{ }^{33}$

Earlier work in this department has shown that $\mathrm{A} \& \mathrm{E}$ is an appropriate place to consider alcohol misuse, with $46 \%$ of detected and referred patients reattending for further help. ${ }^{20}$ An effective screening questionnaire was developed: the one minute PAT. ${ }^{34}$ It was designed with respect to ease of use, speed of administration and the identification of patients at an early stage of alcohol misuse. We have shown previously that once detected, using the PAT, and referred to an alcohol health worker (AHW), 65\% of the $60 \%$ patients who reattended for subsequent counselling reported drinking less at six months, indicating the effectiveness of intervention by an AHW. ${ }^{35}$ In addition, the earlier alcohol misuse is detected the greater is the success of brief intervention. Young patients are often intermittent binge drinkers, ${ }^{36}$ that is, hazardous drinkers as compared with dependent drinkers, whose habits are unrecognised by their general practitioners or other agencies (for example, employers). The potential for $\mathrm{A} \& \mathrm{E}$ to detect young hazardous drinkers has recently been confirmed. ${ }^{37}$ Studies in other environments corroborate the effectiveness of alcohol screening and early intervention. ${ }^{38} 39$

At the start of this study, 26 presenting conditions were listed at the top of the PAT proforma. These had been identified previously $^{34}$ as the most common presentations associated with alcohol misuse and formulated into an easily administered PAT questionnaire (appendix 1). However, the detection rate of alcohol misuse remained low, with a discrepancy between expected and actual AHW referral rates. ${ }^{40}$ This was disappointing given the success rate for ensuing counselling, ${ }^{20}{ }^{35}$ and the spectre of possible medicolegal consequences if alcohol is not considered as a root cause of $\mathrm{A} \& \mathrm{E}^{4041}$ We therefore decided to investigate whether audit/feedback increases PAT use, and whether this has an effect on the numbers of patients referred. The effectiveness of audit in improving the detection of excessive drinkers has been documented previously. ${ }^{42} \mathrm{~A}$ secondary aim was to establish the numbers of each PAT possible complaint presenting to the department with a view to making the PAT a more efficient tool in the hands of junior medical staff.

\section{Methods}

DEFINITIONS AND TERMINOLOGY

(1) The PAT (appendix 1) was used as described previously. ${ }^{34}{ }^{35}$ (2) A patient was described as presenting with a PAT possible condition if (a) a history could be obtained, and (b) a presenting complaint was one listed in the PAT (appendix 1). A patient for whom no history was available (for example, comatose with collateral history not available) or who presented with complaints other than those given in the PAT was deemed non-PAT possible. (3) The minimal criteria for a patient to be coded PAT positive were if (a) they drank more than eight units/day (male) or six units/day (female) on at least one day per week, or (b) the current attendance was related to alcohol. A patient was PAT negative if they replied in the negative to the question concerning units/day and further denied that the current attendance was related to alcohol. A PAT negative coding was derived from the notes if either "PAT negative" was ringed at the top of the clerking proforma or there was documented evidence of an alcohol history being taken, but no indication of an excessive drinking pattern. Thus, in this audit, a PAT possible patient had three possible coded outcomes-(a) PAT not done (= PAT missed) if there was no documentation re alcohol in the notes, (b) PAT negative, or (c) PAT positive.

\section{STUDY DESIGN}

The study period (September to December 1998, when an unchanging team of 13 SHOs was employed) was divided into four $\times$ four week time periods. Each month contained seven "audit days" at four day intervals. During the first (month 1), no feedback was given, nor were the SHOs aware of the audit. During the second and third months, all SHOs were made aware of the audit and received feedback in two forms: (a) individual feedback (to each SHO) concerning all patients in whom a PAT possible condition was identified but no PAT given (that is, PAT missed), and (b) overall feedback at weekly teaching meetings (attended by all SHOs), as to the predominant missed conditions. During the fourth month, only two of the 13 SHOs (CB and JSH) were aware of the audit. These two SHOs performed the audit categorising each patient who fulfilled the inclusion criteria ((1) address (or NFA) in our area, (2) at least 16 years of age, and (3) seen by A\&E SHO) seen in a designated 24 hour period, according to a standard form detailing patient seen, presenting complaints (maximum of three), postcode, PAT possible (Y/N), PAT positivity/negativity/ missed, and referral to AHW. Each patient coded PAT positive could have up to three PAT possible conditions.

\section{DEFINITION OF STANDARD ACCEPTABLE}

Given the possibility of medicolegal consequences raised by Touquet et $a l,{ }^{41}$ the defined standard, at least for the purposes of this study, was PAT use of $100 \%$ for PAT possible complaints. Coupled with the feedback for two months this component of the study fulfilled the criteria for a full audit, ${ }^{43}$ with closure of the audit loop. Given that the PAT represents a formalised approach to obtaining an alcohol history, it was accepted that phrases such as "EtOH-occ." in the notes were coded as PAT 
Table 1 Summary data by month. Number of patient falling into each PAT category, by month. The figures in parentheses are \% of patients presenting with PAT possible condition(s)

\begin{tabular}{lllll}
\hline & \multicolumn{3}{l}{ Month } & \\
\cline { 2 - 5 } & 1 & 2 & 3 & 4 \\
\hline Notes relevant & 402 & 469 & 466 & 424 \\
Notes found & 390 & 465 & 461 & 421 \\
PAT not indicated, not given & 152 & 200 & 171 & 137 \\
EtOH misuse, PAT not possible & 4 & 5 & 4 & 2 \\
PAT negative & $82(35.0)$ & $132(50.8)$ & $183(64.0)$ & $167(59.2)$ \\
PAT positive & $8(3.4)$ & $25(9.6)$ & $41(14.3)$ & $23(8.2)$ \\
PAT possible but not given & $144(61.5)$ & $103(39.6)$ & $62(21.7)$ & $92(32.6)$ \\
\hline
\end{tabular}

negative as, though inadequate in terms of PAT phraseology/completeness of an alcohol history, there was at least written evidence of questioning re alcohol use.

STATISTICS

Conventional $\chi^{2}$ testing was used to compare variables expressed as proportions of PAT possible patients.

\section{Results}

PAT AUDIT

Over the 16 week study, 1761 patients fulfilled the inclusion criteria for the study (address in our area, at least 16 years of age, seen by $A \& E$ $\mathrm{SHO}$ in designated 24 hour period). The notes for $1737(98.6 \%)$ of these were found and analysed. In $15(0.9 \%)$ it was not possible to perform a PAT (whether or not alcohol misuse was suspected). The majority of patients $(1062 ; 61.1 \%)$ presented with at least one condition defined as PAT possible (appendix 1). Table 1 summarises the data by month.

In month 1, PAT uptake was poor with $61.5 \%$ of PAT possible patients having no documentation of alcohol (mis)use in their notes (that is, = PAT missed (ND)). However, when feedback was instituted, uptake was significantly increased $(\mathrm{p}<0.0001)$, with the proportion missed (PAT-ND) decreasing in months 2 and 3 successively (fig 1 ). There was a significant reversal of this effect when feedback was discontinued during month 4 (PAT-ND $=32.6 \% ; \mathrm{p}=0.003)$. The proportions of PAT possible patients identified as PAT positive and PAT negative also increased

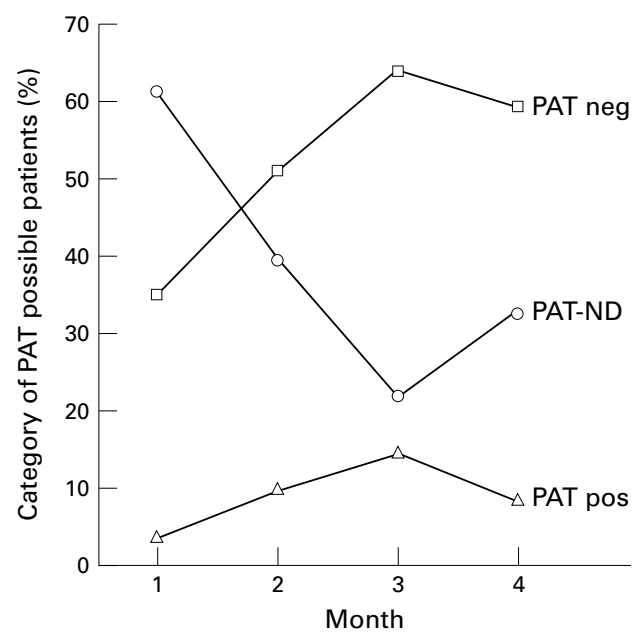

Figure 1 Categorisation of PAT possible patients according to month. PAT-ND = PAT possible but not done.

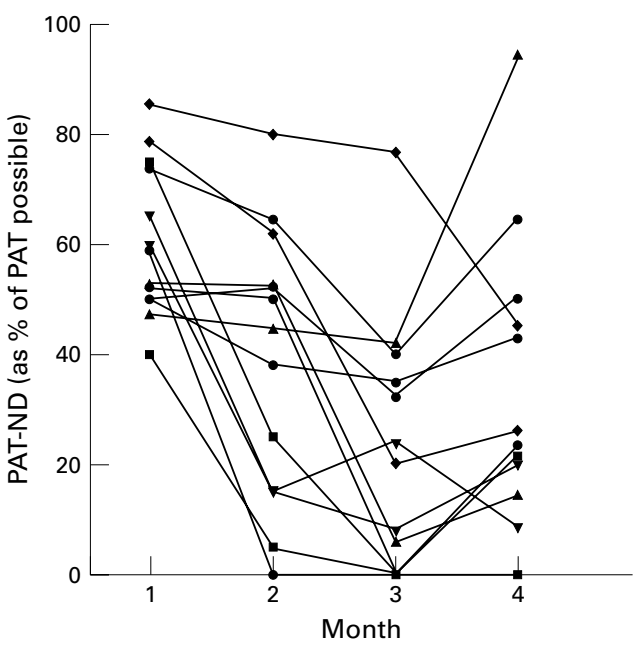

Figure 2 Individual SHO performances showing PAT-ND (PAT possible patients with PATs not done) as a percentage of their total PAT possible load, according to month.

with feedback. Of PAT possible patients, 35\% were identified as PAT negative in month 1 compared with $50.8 \%, 64.0 \%$ and $59.2 \%$ in months 2, 3 and 4, respectively. Only 3.4\% were identified as PAT positive in month 1 , compared with $9.6 \%, 14.3 \%$ and $8.2 \%$ in months 2, 3 and 4 (table 1).

Data concerning \%PAT missed for each SHO are given in figure 2. Though audit and feedback improved SHO performance, there was nevertheless marked SHO variability. Therefore on any given day, results are likely to be influenced not only by case mix but also by "SHO mix".

Figure 3 shows the increase in number of patients identified as PAT positive when analysis was augmented by feedback, and the reversal of this trend when feedback was withdrawn. It also shows the increasing numbers of these patients referred (black bars).

\section{PREVALENCE OF PAT POSSIBLE COMPLAINTS}

The most common PAT possible conditions were: fall $>$ non-specific gastrointestinal problem $>$ chest pain $>$ head injury $>$ "unwell" = other (non-domestic) assault. These six complaints accounted for $63.6 \%$ of all the PAT

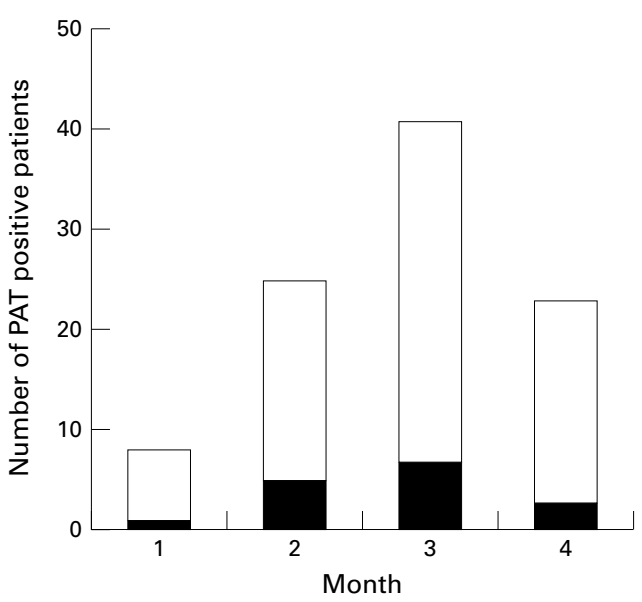

Figure 3 Number of PAT positive patients according to month. Black area shows number accepting $A H W$ referral. 
Table 2 PAT positive yield according to modified complaint groupings

\begin{tabular}{lll}
\hline Complaint by modified grouping & $\begin{array}{l}\text { PAT positive/(PAT } \\
\text { positive }+ \text { PAT } \\
\text { negative) as \% }\end{array}$ & $\begin{array}{l}\text { PAT positive/(PAT } \\
\text { possible) as \% }\end{array}$ \\
\hline Fall & 19.5 & 9.7 \\
Collapse (including "fit", "blackout") & 23.2 & 16 \\
Head injury & 24.1 & 14 \\
Assault (including "domestic violence", "other") & 30.2 & 17.1 \\
Non-specific gastrointestinal & 7.5 & 5.2 \\
Unwell & 18.0 & 13.2 \\
Psychiatric ("depression", "overdose", "confusion") & 29.5 & 13.3 \\
Cardiac (including "palpitations", "chest pain") & 5.2 & 3.0 \\
Self neglect & 50 & 31.3 \\
Repeat attender & 66.7 & 40 \\
\hline
\end{tabular}

possible conditions. Despite re-iteration at feedback of the importance of these PAT possible conditions, they continued to form the bulk of all classes of PAT possible patients, including the PAT missed group.

One weakness of the original PAT (appendix 1) was the large number (26) of PAT possible complaints. It was judged that these could be regrouped into a smaller number of sections. Of the 139 PAT positive patients identified during the study, $77.0 \%$ (107) were accounted for by these "top 10" complaints/groupings arranged as follows: fall > collapse (including "fit", "blackout") > head (including facial) injury > assault (comprised of "domestic assault" and "other") > non-specific gastrointestinal $>$ unwell $>$ psychiatric (including "depression", "overdose", "confusion") > cardiac (including palpitations, chest pain) $>$ self neglect $>$ repeat attendance.

Data in table 2 show the PAT positive yield expressed as ratios: (1) (PAT positive/(PAT positive + PAT negative)), and (2) (PAT positive/PAT possible). Column 1 therefore shows the yield of PAT positives as $\%$, wherein the denominator is the number of patients with this presenting complaint that were actually asked about alcohol. Column 2 shows the yield of PAT positives, with the number of PAT possible as denominator. A marked discrepancy therefore, between the two columns for any category indicates that doctors are poor at asking about alcohol for this condition (for example, falls or psychiatric).

\section{Discussion}

This study shows the value of ongoing audit with feedback in detection of alcohol misuse within A\&E. This process significantly increased PAT use and the identification of PAT positive patients while withdrawal of feedback resulted in decreased PAT use and number of PAT positive patients detected (figs $1,2,3$ ). Not surprisingly, there was large variation between SHOs in the number of completed questionnaires over the four month study period, with a trend for fewer reports to be completed at night (when the department is generally busier) than during the day.

Several modifications have been made to the PAT to make it faster to complete and more user friendly. The PAT possible complaints have been reformulated in a revised PAT with 10 PAT possible complaints (fall, collapse (including fits), head injury (especially facial), assault, non-specific gastrointestinal, "unwell", psychiatric (including overdose), cardiac (including palpitations), self neglect, repeat attender) as compared with 26 (appendix 1). This "top 10" will be printed in future editions of our A\&E record to serve as a further reminder to the clinician. The second part of question 2 on the PAT has been removed. All patients who were PAT positive on this criterion, were PAT positive on the basis of question 3 as well- that is, this section of question 2 conferred no additional discriminatory power. Furthermore, it is proposed to make ongoing audit a feature of PAT practice in the department, as withdrawal of feedback caused a reversal of the improvement of PAT uptake. Our study has confirmed that the "top 10" PAT possible conditions seen in central London are similar to the predominant conditions highlighted in studies from Baltimore ${ }^{23}$ and Los Angeles. ${ }^{11}$

Table 2 shows derivative data of ratios for the PAT positive yield of each complaint category. The ranking of each category was broadly consistent between the two columns. Repeat attenders were the most likely to be found PAT positive. Conversely and surprisingly, "cardiac" presentations (comprising chest pain, palpitations) had a poor PAT positive yield. Alcohol is known to cause myopathic changes ${ }^{44}$ and cardiac arrhythmias, but the resulting clinical problems are not common. Some categories such as "fall" are common presentations in $\mathrm{A} \& \mathrm{E}$ frequently associated with alcohol misuse, but the opportunity for screening for alcohol misuse is still often missed. Interestingly, although 51 complaints pertained to road traffic accidents, no such patient admitted to being PAT positive. Legislation for drinking and driving is an example of a recent change in attitudes and practice. ${ }^{45}$

Patients were asked, in question 1 of the PAT, their maximum daily alcohol consumption, which may underestimate the actual amount drunk. The values of six units for women and eight units for men that we used as a cut off are twice the recommended daily maximum, ${ }^{46}$ but were chosen empirically as alcohol intake above these values is associated with an increased incidence of facial injuries. ${ }^{6} 8$ Our methodology did not review blood alcohol concentrations. Interestingly, Carrigan et $a l^{47}$ demonstrated a significantly higher prevalence for alcohol intoxication in the non-RTA trauma group than the RTA trauma group. We suggest that where a patient is unable to answer questions concerning possible alcohol misuse, then a blood alcohol level should be requested. This should also apply to the non-traumatic conditions listed in the "top 10".

Despite the generally positive attitudes of A\&E medical professionals towards detecting alcohol misuse, and the issue of medicolegal liability if alcohol is ignored as a possible cause for certain complaints, ${ }^{31}{ }^{41}$ there are few examples of alcohol treatment facilities being integrated with A\&E departments. ${ }^{15}$ Indeed, at present we know of only three other UK A\&E departments that incorporate AHWs (Hope Hospital, Manchester; Royal Liverpool University Hospital; North Tees General Hospital, 
Stockton on Tees). We believe this should become universal practice, to minimise missed opportunities for early intervention in the treatment of alcohol misuse.

Part of the role of A\&E doctors and nurses is to detect alcohol misuse in a non-judgemental manner, and to encourage the patient to recognise that they have a problem. In this way, patients may become receptive to the idea of self help and want to be referred to an AHW. The role of the trained AHW is to provide counselling. A lack of definition of such roles is, in our view, why other studies have suggested that $\mathrm{A} \& \mathrm{E}$ is a difficult location to motivate drinking patients to alter their habits. ${ }^{48-50}$

Nationally, it is time to confront the apathy with which the health service in general and $\mathrm{A} \& \mathrm{E}$ in particular regard the detection and treatment of alcohol misuse. ${ }^{51}$ The fact that more patients present to A\&E with a PAT possible than a non-PAT possible condition means that for the "top 10" presentations, the question every doctor should ask themselves is not so much "Should I ask about alcohol?", but "Can I afford not to ask about alcohol?".

We gratefully acknowledge the cooperation and forbearance of all SHOs in "Team 25" and our AHWs including Phillip May, Adrian Brown, BKCW Mental Health Trust. In addition we thank Mr A Duffy and Ms S Wright for access to their internal audit of 1997; Professor J A Henry, Dr T Cha G T Smith for

Conflicts of interest: none.

Addendum

Updated PATs (PAT 2001) available from R Touquet.

1 Davidson P, Koziol-McLain J, Harrison L, et al. Intoxicated ED patients: a 5-year follow-up of morbidity and mortality. Ann Emerg Med 1997:30:593-7

2 Moore S. Booze. Britain's real drug crisis. Independent. Friday Review 7 August, 1998:1.

Chang G, Astrachan BM. The emergency department surveillance of alcohol intoxication after motor vehicle accidents. $¥ A M A$ 1988;260:2533-6

4 Paton A, ed. $A B C$ of alcohol. London: BMJ Publishing Group, 1994

5 Paton A. Barriers to education about alcohol. $7 \mathrm{R}$ Soc Med 1992;85:476-8

6 Shepherd J, Brickley M. The relationship between alcohol intoxication, stressors and injury in urban violence. British fournal of Criminology 1996;36:546-66.

7 Brismar B, Bergman B. The significance of alcohol for violence and accidents. Alcohol Clin Exp Res 1998;22 (suppl 7):299-306S

8 Shepherd J. Emergency room research on links between alcohol and violent injury. Addiction 1998:93:1261-3

9 Macdonald S, Wells S, Giesbrecht N, et al. Demographic and substance use factors related to violent and accidenta injuries: results from an emergency room study. Drug Alcohol Depend 1999;55:53-61.

10 Smith GS, Branag CC, Miller TR. Fatal nontraffic injuries involving alcohol: a metaanalysis. Ann Emerg Med 1999;33 659-68

11 Whiteman PJ, Hoffman RS, Goldfrank LR. Alcoholism in the emergency department: an epidemiologic study. Acad Emerg Med 2000;7:14-20

12 Rowland N, Maynard A, Beveridge A, et al. Doctors have no time for alcohol screening. BMf 1987;295:95-6

13 Rowland N, Maynard A, Kennedy P, et al. Doctors and alcohol screening - the gap between attitudes and action. Health Educ $\mathcal{f} 1988 ; 47: 133-6$.

14 Walsh RA, Sanson-Fisher RW, Reid ALA. Australian developments in medical education about alcohol. $\mathcal{F} R$ Soc Med 1993;86:313-14.

15 Hadfield JM, Duxbury R, McMahon G, et al. Brief interventions for alcohol in the emergency department: a
purchaser's perspective. [Abstract]. F Accid Emerg Med purchaser's pers

16 Batel P, Pessione F, Bouvier A-M, et al. Prompting alcoholics to be referred to an alcohol clinic: the effectiveness of a simple letter. Addition 1995;90:811-14.

17 Lockhart T. Problem drinkers in Accident and Emergency: health promotion initiatives. Accid Emerg Nurs 1997;5:1621.

18 Herring R, Thom B. A\&E nurses and alcohol-related attendances. Nursing Times 1999;95:59-62.
19 National Audit Office. NHS Accident and Emergency Departments in England. London: HMSO (158), 1992.

20 Green M, Setchell J, Hames P, et al. Management of alcohol abusing patients in accident and emergency departments. $\mathcal{F}$ $R$ Soc Med 1993;86:393-5.

21 Yates DW, Hadfield JM, Peters K. Alcohol consumption of patients attending two accident and emergency departments in north-west England. $\mathcal{F} R$ Soc Med 1987a; 80:486-

22 Yates DW, Hadfield JM, Peters K. The detection of problem drinkers in the accident and emergency department. $B r F$ Addiction 1987b;82:163-7.

23 Li G, Keyl PM, Rothman R, et al. Epidemiology of alcoholrelated emergency department visits. Acad Emerg Med 1998;5:788-95

24 Stimson GV, Hayden D. Drugs: The state of the region, 1993substance use and 'The Health of the Nation'. London: The Centre for Research on Drugs and Health behaviour, 1993.

25 D'Onofrio G, Bernstein E, Bernstein J, et al. Patients with alcohol problems in the emergency department, part 1: improving detection. Acad Emerg Med 1998;5:1200-9.

26 D’Onofrio G, Bernstein E, Bernstein J, et al. Patients with alcohol problems in the emergency department, part 2: intervention and referral. Acad Emerg Med 1998;5:121017.

27 Gentilello LM, Rivara FP, Donovan DM, et al. Alcohol interventions in a trauma center as a means of reducing the risk of injury recurrence. Ann Surg 1999;230:473-83.

28 Williams RJH, Hittenger R, Glazer G. Resource implications of head injuries in acute surgical units. F $R$ Soc Med $1994 ; 87: 83-6$.

29 Ferguson RK, Sorgal IN, Pentland B. Thiamine deficiency in head injury: a missed insult? Alcohol Alcohol 1997;32: 493-500.

30 Cherpitel CJ. Alcohol and injuries: a review of international emergency room studies. Addiction 1993;88:923-37.

31 Waller S, Thom B, Harris S, et al. Perceptions of alcohol-related attendances in accident and emergency departments in England: a national survey. Alcohol Alcohol 1998;33:354-61.

32 Hutchison IL, Magennis P, Sheperd JP, et al. The BAOMS United Kingdom Survey of facial injuries Part 1: aetiology and the association with alcohol consumption. $\mathrm{Br} F$ Oral Maxillofac Surg 1998:36:3-13.

33 Brooker C, Peters J, McCabe C, et al. The views of nurses to the conduct of a randomised controlled trial of problem drinkers in an accident and emergency department. Int $\mathcal{F}$ Nurs Stud 1999;36:33-9.

34 Smith SGT, Touquet R, Wright S, et al. Detection of alcohol misusing patients in accident and emergency departments: the Paddington alcohol test (PAT). F Accid Emerg Med 1996;13:308-12.

35 Wright S, Moran L, Meyrick M, et al. Intervention by an alcohol health worker in an accident and emergency department. Alcohol Alcohol 1998;33:651-6.

36 Murgraff V, Parrott A, Bennett P. Risky single-occasion drinking amongst young people, definition, correlates, policy, and intervention: a broad overview of research findings. Alcohol Alcohol 1999;34:3-14.

37 Thom B, Herring R, Judd A. Identifying alcohol-related harm in young drinkers:the role of A\&E departments. Alcohol Alcohol 1999;34:910-15.

38 Smith AJ, Shepherd JP, Hodgson RJ. Brief interventions for patients with alcohol-related trauma. Br f Oral Maxillofacial Surg 1998;36:408-15.

39 Perry M. Alcohol screening and early interventions in the medical setting. Alcoholism 1999;18:3-4.

40 Touquet R, Wright S, Brown A. Detection of alcohol misuse using the "Paddington alcohol test", with subsequent counselling by an alcohol healthworker: 1998 onwards. [Abstract]. F Accid Emerg Med 1998;15:412.

41 Touquet R, Fothergill J, Henry JA, et al. Accident and emergency departments. In: Powers MJ, Harris NH, eds. Clinical negligence. 3rd ed. London: Butterworths, 2000.

42 Dent T, Shepherd R, London M, et al. Education and audit can improve the identification of excessive drinkers among medical inpatients. Health Trends 1995;27:92-7.

43 Lawrence M, Griew K, Derry J, et al. Auditing audit: use and development of the Oxfordshire medical audit advisory group rating system. BM7 1994;309:513-16.

44 Preedy VR, Peters TJ. Alcohol and muscle disease. $f R$ Soc Med 1993;87:188-90.

45 Morgan G. Drinking and driving legislation: the need for targetted prevention programmes. Alcoholism 1999;18:1-3.

46 Chief Medical Officer. Sensible drinking. CMO's update. London: Department of Health, 1996:4.

47 Carrigan TD, Field H, Illingworth RN, et al. Toxicological screening in trauma. F Accid Emerg Med 2000;17:33-7.

48 Robertson CE, Little K, Smith H, et al. The impact of alcohol on the acute hospital service: patient presentation, admission and the perception of alcohol use in such groups. Alcohol Alcohol 1989;24:405-8.

49 Peters J, Brooker C, McCabe C, et al. Problems encountered with opportunistic screening for alcohol related problems in patients attending an accident and emergency department. Addiction 1998;93:589-94.

50 Templeton J. The organisation of trauma services in the UK. Ann R Coll Surg Engl 2000;82:49-52.

51 Hargarten SW. Alcohol-related injuries: do we really need more proof? Ann Emerg Med 1999;33:699-701. 
Appendix 1

The Paddington Alcohol Test (study format)

The one minute

\section{'PADDINGTON ALCOHOL TEST'}

(P.A.T.) n.b. mark 'page 3' A\&E card

Complete for ALL patients where any INDICATION of ALCOHOL misuse: DOMESTIC VIOLENCE, other ASSAULT, head esp. facial injury, fall, PEDESTRIAN RTA, other RTA, nonspecific G.I. problem, "unwell", fit, blackout, collapse, insomnia, hypo/h'erglyc'ia, sweating, palpitations, chest pain, gout, rashes, depression, overdose; note REPEAT attendance (perhaps unexplained symptoms) + DELAYED attendance $>4 \mathrm{hrs}$ (perhaps intoxicated at 'incident'). N.B. The Elderly presenting with: falls, confusion, incontinence and self-neglect.

Please circle most specific triggers; maximum 3 circles e.g. DOMESTIC., head i., REPEAT.

\section{DATE:}

\section{PATIENT IDENTIFICATION STICKER:}

After Dealing with patient's 'agenda,' i.e. patient's reason for attendance:-

1. 'Quite a number of people have times when they drink more than usual; what is the most you will drink in any one day?, (Units, 8 gms alc., for pub measures are in brackets; home measures often $\times 3$ !)

Beer/lager/cider
Strong Beer/lager/cider
Wine
Fortified Wine (Sherry, Martini)
Spirits (Gin, Whisky, Vodka)
Home measures YES/NO

$\begin{array}{lll}\text { _Pints (2) } & \text { _Cans (1.5) } & \text { total Units/day } \\ \text { _Pints (5) } & \text { _Cans (4) } & \\ \text { _Glasses (1.5) } & \text { _Bottles (9) } & \\ \text { _Glasses (1) } & \text { _Bottles (12) } & \\ \text { _Singles (1) } & \text { _Bottles (30) }\end{array}$

2. If this is more than 8 units/day for a man, or 6 units/day for a woman, does this happen

$$
\begin{aligned}
& \text { : Once a week or more = YES: PAT +ve (? Pabrine } \times) \\
& \text { or } \\
& \text { : Between once a month and once a week? = YES: PAT +ve } \\
& \text { or } \\
& \text { : Less frequent? } \quad=\quad \text { ?PAT -neg (? as trumped by Quest. 3) }
\end{aligned}
$$

3. 'Do you feel your current attendance in A\&E is related to alcohol?'

YES(PAT +ve)/NO

If PAT +ve: 'Would you like to see our Health Worker?'

YES/NO

If $\underline{\text { YES: }}$ give Alcohol Advice Card with appointment for next Review Clinic + book appt.

If NO: 1. Still give patient Alcohol Advice Card: patient may change mind later and return. 2. Still complete PAT: place in notes for reinforcement if patient reattends.

3. Still mark A\&E notes, p.3: PAT 'POS', Referred AHW 'NO', to alert staff.

4. Still inform G.P. in A\&E discharge letter: no.11 letter if alcohol main problem.

If to be admitted, note in AHW book \& state ward if known

SIGNATURE:

NAME STAMP: 